\title{
Tratamiento intervencionista en tromboembolia pulmonar
}

\author{
Jaime Eduardo Morales-Blanhir, $\bowtie$ Jorge Daniel Sierra-Lara-Martínez, \\ María de Jesús Rosas-Romero
}

\author{
Instituto Nacional de Ciencias Médicas y Nutrición Salvador Zubirán, \\ Ciudad de México. \\ Trabajo recibido: 10-IV-2013; aceptado: 11-VI-2013
}

\begin{abstract}
RESUMEN. La tromboembolia pulmonar es una enfermedad común y una de las principales causas de muerte. El tromboembolismo pulmonar masivo requiere un abordaje e intervención terapéutica agresivos y oportunos. La tromboembolia pulmonar aguda asociada a inestabilidad hemodinámica (choque o hipotensión arterial) tiene un riesgo alto de mortalidad (25-65\%) en las primeras horas, y hasta el $10 \%$ de riesgo de progresar a choque cardiogénico; se estima que hasta en el $50 \%$ de los casos esta se asocia a tromboembolia pulmonar recurrente. El tratamiento que con base en la evidencia ha demostrado mejoría clínica y disminución en el riesgo de mortalidad es la anticoagulación. Sin embargo, existen condiciones clínicas en las que el inicio de esta terapia puede estar contraindicado; es decir, deberán considerarse otras opciones terapéuticas. Al presente, no existe evidencia concreta en base a estudios clínicos, de la utilidad de las opciones alternas que sean comparables con la anticoagulación, en el tratamiento de la tromboembolia pulmonar; se discuten las distintas opciones disponibles en tromboembolia pulmonar aguda.
\end{abstract}

Palabras clave: Tromboembolismo pulmonar, circulación pulmonar, embolismo pulmonar masivo, terapia asistida por catéter, terapia endovascular.

\begin{abstract}
Pulmonary embolism is a common cause of death. Massive pulmonary thromboembolism requires an aggressive and timely therapeutic approach. Acute pulmonary embolism associated with hemodynamic instability (shock or hypotension) has a high risk of mortality in the early hours (25-65\%) and $10 \%$ risk of progressing to cardiogenic shock; an estimate of up to $50 \%$ of cases are associated with recurrent pulmonary thromboembolism. The treatment based on evidence that has shown clinical improvement and decreased risk of mortality is anticoagulation. However, there are clinical conditions in which the use of this therapy may be contraindicated or can be detrimental to the patient's clinical status and therefore other therapeutic options should be considered. At present there is no concrete evidence based on clinical trials for the use of alternative options for the treatment of pulmonary embolism that are comparable with anticoagulation. We will discuss the different options available in acute pulmonary embolism.
\end{abstract}

Key words: Pulmonary thromboembolism, pulmonary circulation, massive pulmonary embolism, catheter-directed interventions, endovascular therapy.

\section{INTRODUCCIÓN}

La enfermedad tromboembólica pulmonar comprende a la tromboembolia pulmonar (TEP) y la trombosis venosa profunda (TVP). La TEP es una entidad común con una elevada tasa de morbimortalidad; ${ }^{1}$ ocupa la tercera causa de muerte cardiovascular, precedida por la cardiopatía isquémica y la enfermedad vascular cerebral. ${ }^{2-4}$ Los principales factores de riesgo son: antecedente de intervenciones quirúrgicas, trauma, insuficiencia cardíaca congestiva, cáncer, embarazo, uso de anticonceptivos orales, obesidad y edad avanzada.

El inicio de la terapia en forma oportuna disminuye el riesgo de mortalidad en un $<10 \% .{ }^{5}$ Las opciones terapéuticas tradicionales son la anticoagulación, la trombólisis sistémica y la trombectomía quirúrgica.
Recién se han introducido varios procedimientos de invasión mínima, como trombólisis asistida por catéter, embolectomía percutánea, técnicas de fragmentación percutánea del trombo con una variedad de dispositivos, colocación de un stent de arteria pulmonar, o el uso de dos o más de estas técnicas. ${ }^{5}$

El resultado favorable en el tratamiento de la TEP requiere la oportuna estratificación de riesgo, así como el uso de técnicas y dispositivos adecuados. Las indicaciones para intervención agresiva en TEP masiva son las siguientes (por lo menos un indicador presente): $: 5$

1. Hipotensión arterial (tensión arterial sistémica $<90$ $\mathrm{mmHg}$ o disminución de $>40 \mathrm{mmHg}$ ).

2. Choque cardiogénico. 
3. Hallazgos por ecocardiografía que indiquen disfunción ventricular derecha o hipertensión arterial pulmonar.

4. Diagnóstico de hipertensión arterial pulmonar $>20$ $\mathrm{mmHg}$ en presencia de presión parcial de oclusión de arteria pulmonar normal.

5. Gradiente alvéolo-arterial $\left(\mathrm{GA}-\mathrm{aO}_{2}\right)$ amplio (> 50 $\mathrm{mmHg}$ ).

6. Contraindicación para anticoagulación o terapia trombolítica.

\section{Filtro de vena cava inferior}

El filtro de vena cava inferior ( $\mathrm{VCl}$ ) funciona como un protector que permite el paso de la sangre mientras se encarga de bloquear y fragmentar el paso de émbolos provenientes de extremidades inferiores, evitando su llegada a la circulación pulmonar. ${ }^{7}$

Aproximadamente $15 \%$ de los pacientes con diagnóstico de TVP se someten a la colocación de filtro de $\mathrm{VCl}$. Las indicaciones se dividen en dos grupos: Absolutas: cuando existe contraindicación absoluta

Tabla 1. Indicaciones de filtro de vena cava inferior.

\section{Indicaciones aceptadas:}

- Contraindicación absoluta para anticoagulación terapéutica o complicaciones con sangrado activo.

- Falla de la anticoagulación en el contexto de trombosis venosa profunda proximal.

Indicaciones de uso controversial:

- Trombosis venosa proximal en paciente con pobre reserva cardiopulmonar.

- Tromboembolia pulmonar aguda masiva y/o hipertensión arterial pulmonar crónica tromboembólica.

- Trombosis venosa profunda iliocaval.

- Profilaxis (trauma mayor en casos que exista contraindicación para anticoagulación profiláctica).

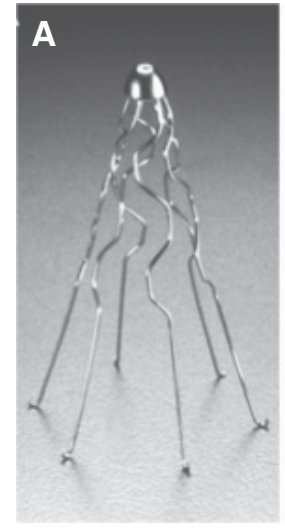

B

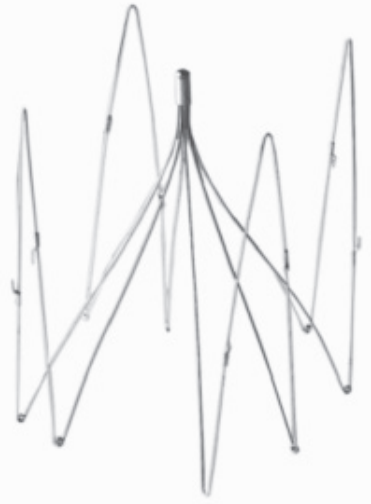

para anticoagulación terapéutica, sangrado activo, hipertensión pulmonar tromboembólica crónica, falla de la anticoagulación en el contexto de trombosis venosa profunda proximal. Relativas: trombosis venosa iliocaval, dificultad para el mantenimiento de anticoagulación terapéutica, pobre adherencia al tratamiento.

En ocasiones, los filtros de VCI son colocados en el contexto de TEP masiva (TEP asociada a inestabilidad hemodinámica), en especial para pacientes con riesgo de mortalidad con contraindicación para la terapia trombolítica, o en aquellos en los que la vasculatura pulmonar se encuentra comprometida por un evento de TEP previo, de forma tal que un segundo evento no sería bien tolerado clínica ni hemodinámicamente (v.gr., TEP aguda masiva y/o hipertensión arterial pulmonar crónica tromboembólica) ${ }^{5,8,9}$ (tabla 1).

\section{Colocación}

Se realiza de manera percutánea vía femoral o yugular; por lo general, se fijan en vena cava infrarrenal. Se prefiere el abordaje yugular por un menor grado de complicaciones en relación con la colocación vía femoral (trombosis femoral). Se pueden colocar filtros de $\mathrm{VCl}$ suprarrenales cuando existe trombosis de las arterias renales, trombosis en $\mathrm{VCl}$ con extensión renal y trombosis $\mathrm{VCl}$ infrarrenal que no permita manipulación para la colocación del mismo. ${ }^{9}$

\section{Filtros de vena cava inferior permanentes y no permanentes}

Con el advenimiento de los filtros de VCI no permanentes se ha dado una mayor colocación de los mismos; empero, no existen recomendaciones basadas en evidencia acerca de su uso y la preferencia de uno sobre el otro, ${ }^{5} v$. gr. figura 1.

El informe más significativo procede del registro ICOPER (del inglés International Cooperative Pulmo-

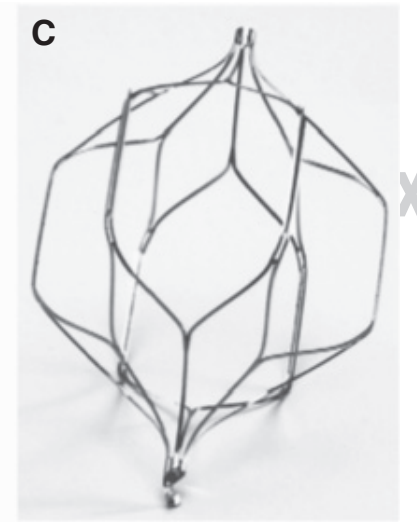

Figura 1.

Ejemplos de filtros de $\mathrm{VCl}$ : A) filtro original Greenfield $64 \mathrm{fr}$; B) filtro vena Tech; C) filtro rescatable Optease, con sitio de fijación con orientación caudal para retracción vía femoral (2003).

Modificado de: Tapson VF. Clin Chest Med 2010;31:771-781. (Referencia 12). 
nary Embolism Registry) que incluyó 2,284 pacientes con TEP submasiva y 108 pacientes con TEP masiva (definido por tensión arterial sistólica $<90 \mathrm{mmHg}$ ). Únicamente 11 de 108 pacientes con TEP masiva recibieron como tratamiento la inserción del filtro de $\mathrm{VCl}$. Ninguno presentó enfermedad tromboembólica recurrente; más aún, 10 de 11 pacientes sobrevivieron a un seguimiento médico de 90 días. Con base en estos datos, los autores concluyen que la colocación del filtro de $\mathrm{VCl}$ en pacientes con TEP masiva incide en reducir la mortalidad a 90 días (HR 0.12, 95\% IC 0.02-0.85). ${ }^{8,10}$

El filtro de $\mathrm{VCl}$ permanente se utiliza en pacientes con TVP con contraindicación para anticoagulación a largo término. ${ }^{5-8} \mathrm{El}$ filtro de $\mathrm{VCl}$ no permanente se utiliza en pacientes con TVP y contraindicación para anticoagulación a corto plazo o que presenten un daño cardiopulmonar previo. Con el fin de evitar complicaciones a largo plazo asociadas a dispositivos no permanentes, deben realizarse evaluaciones periódicas de 3 a 12 meses posteriores a la colocación del filtro, de acuerdo con las especificaciones del fabricante de los dispositivos mencionados. ${ }^{8}$ Previa evaluación y retiro del filtro de $\mathrm{VCl}$, se deberá realizar venografía con el fin de identificar trombos asociados que impliquen dejarlo a permanencia. ${ }^{8}$

En las recomendaciones emitidas por el ACCP (del inglés American College of Chest Physicians) para el tratamiento y prevención de trombosis, se sugiere que en pacientes con TEP a quienes se les colocó filtro de $\mathrm{VCl}$ como terapia alternativa, el inicio de terapia antitrombótica convencional se realiza una vez resuelto el riesgo de sangrado; en el caso de filtros de $\mathrm{VCl}$ permanentes no se considera indicación para terapia antitrombótica a largo plazo. ${ }^{11}$

\section{Evidencia clínica}

Al presente, no existen estudios bien diseñados que comparen directamente las diferencias entre los filtros de $\mathrm{VCl}$ permanentes y no permanentes. ${ }^{8,12}$

En el estudio para la prevención de riesgo de TEP mediante interrupción de vena cava (PREPIC study, del francés Prevention du Risque d’Embolie Pulmonaire par Interruption Cave), por asignación aleatoria se estudiaron 400 pacientes con diagnóstico de TVP y alto riesgo para TEP para recibir anticoagulación con heparina no fraccionada (HNF) versus heparina de bajo peso molecular (HBPM) con o sin colocación de filtro de $\mathrm{VCl}^{8,13}$ Se evaluó en término de eficacia el diagnóstico confirmado de TEP a un seguimiento de 8 años. Además, se evaluaron los siguientes objetivos: TVP recurrente, sangrado mayor y muerte a los 12 días, 2 años y 8 años. Se demostró que los filtros de $\mathrm{VCl}$ reducen la incidencia de TEP recurrente a los 12 días ( 1.1 versus $4.8 \% p=0.03$ ), a 8 años ( 6.5 versus $15.1 \%$ $p=0.008$ ); sin embargo, se identificó que la presencia de filtros de $\mathrm{VCl}$ se asocia a una mayor incidencia para TVP recurrente a los 2 años (20.8 versus $11.6 \%$ $p=0.02$ ). Sin demostrar diferencia estadísticamente significativa para sangrados mayores, insuficiencia venosa crónica postrombótica o muerte, y con ningún efecto sobre la mortalidad global. . $^{8,13}$

En un estudio observacional realizado por White et al., se demostró, al igual que en el PREPIC, que la colocación de filtro de $\mathrm{VCl}$ redujo la incidencia de rehospitalización por TEP, aunque también se identificó un incremento en la incidencia de rehospitalización secundaria a TVP en pacientes que de manera inicial habían presentado TEP (RR 6.72, 95\% IC, 3.61-12.49). ${ }^{9}$

\section{Complicaciones}

Se han reportado algunas complicaciones asociadas con este procedimiento. En general, se dividen en tempranas o tardías con relación al tiempo de colocación del filtro. La tasa de mortalidad es del $0.1 \%$. Las complicaciones tempranas son: trombosis venosa (vena femoral común, $8.5 \%)$, posición inadecuada (1.3\%), neumotórax $(0.02 \%)$, hematoma $(0.06 \%)$, punción arterial carotídea $(0.04 \%)$, fístula arteriovenosa $(0.02 \%)$. Se puede reducir el riesgo mediante el uso racional de punciones guiadas por ultrasonido o fluoroscopia. Las complicaciones tardías incluyen: TVP recurrente (21\%), trombosis de $\mathrm{VCl}(2-10 \%)$ y migración de filtro $(0.3 \%)^{7,8,12}$

\section{Trombectomía quirúrgica}

La TEP masiva representa un estado de inestabilidad hemodinámica que requiere de estrategias invasivas para disminuir la repercusión a la circulación pulmonar. Anteriormente, en pacientes con pobre respuesta clínica o con contraindicación absoluta a las terapias médicas o trombolíticas, la única opción disponible consistía en realizar trombectomía quirúrgica con esternotomía y extracción del trombo de arteria pulmonar principal; sin embargo, este procedimiento se asocia a una gran morbilimortalidad en relación con el procedimiento en sitios con poca experiencia. ${ }^{6,7}$

En el contexto de pacientes con TEP e inestabilidad hemodinámica, especialmente asociado a dilatación de ventrículo derecho o hipocinesia, la ACCP recomienda que la embolectomía quirúrgica se considere en pacientes con TEP de alto riesgo, en quienes la terapia trombolítica se encuentre contraindicada por el riesgo de sangrado o el paciente no tolere el tiempo suficiente 
para que la terapia trombolítica sea efectiva. ${ }^{11}$ Con esta estrategia se puede evitar la muerte en pacientes con obstrucción de más del $50 \%$ de la arteria pulmonar, con mortalidad de $85 \%$ en las primeras 6 horas. ${ }^{5}$

En 1994 Gulba et al. reportaron 37 casos con el diagnóstico de ser sometidos a tratamiento basado en infusión de rt-PA (activador tisular del plasminógeno recombinante) o trombectomía quirúrgica. La tasa de éxito del tratamiento quirúrgico fue superior (85 versus $75 \%$ ), así como menor frecuencia de mortalidad (23 versus $33 \%$ ). La limitante principal es que los datos derivan de un reporte de casos y los resultados no pueden generalizarse, dado que carecen de validez externa. 5,14

Debido a la complejidad de la entidad, no existen estudios que realmente demuestren las ventajas y beneficios de este abordaje; se requiere de una decisión rápida por la condición clínica de los pacientes en cuestión y de un equipo de expertos en abordajes quirúrgicos cardiopulmonares. Al parecer, los resultados son mucho más alentadores en centros donde la experiencia clínica es mayor; ${ }^{11,12}$ con una supervivencia del $89 \%$, atribuida principalmente a la experiencia del equipo quirúrgico, diagnóstico oportuno y adecuada selección de pacientes. ${ }^{15}$

\section{Trombectomía percutánea mecánica}

La trombectomía percutánea mecánica se refiere al uso de dispositivos asistidos por catéteres; a través de éstos se remueve el trombo de la circulación pulmonar por aspiración, fragmentación o maceración ${ }^{8,16}$ (tabla 2 y figura 2).

Las metas de la intervención asistida por catéter incluyen: a) rápida reducción de la presión de la arteria pulmonar, resistencias pulmonares vasculares y presión ventricular derecha; b) mejorar perfusión sistémica; c) facilitar recuperación de ventrículo derecho. ${ }^{8}$

Actualmente, como sucede con la trombólisis sistémica y trombectomía quirúrgica, no existen

Tabla 2. Trombectomía asistida por catéter.

\begin{tabular}{ll}
\hline Técnica & Ejemplos \\
\hline Aspiración & Dispositivo de aspiración \\
Fragmentación & Greenfield \\
& Catéter rotatorio «cola de \\
cochino» \\
Hidrodinámica-reolíticos & Amplatz \\
& Aspirex \\
& Angiojet \\
& Hydrolyser \\
Trombólisis local & tPa (Alteplase) \\
& Urokinasa \\
\hline
\end{tabular}

evidencias basadas en estudios confiables para realizar recomendaciones del uso de esta terapia. Se ha demostrado desde hace más de 20 años que la infusión de un agente trombolítico directamente a la arteria pulmonar no ofrece mayor beneficio sobre infusión sistémica. ${ }^{12,17}$

Acorde con las estrategias de tratamiento antitrombótico no farmacológico emitidas por la ACCP y la Sociedad Europea de Cardiología, en pacientes con TEP aguda e inestabilidad hemodinámica que tienen contraindicación o fallo en la trombólisis o choque hemodinámico con riesgo de muerte, se recomienda la trombectomía no quirúrgica (asistida por catéter), siempre y cuando sea en un centro con experiencia y equipo médico necesario para su ejecución. .11 $^{3,11}$

La búsqueda de un dispositivo ideal para el tratamiento asistido ha llevado a la producción de múltiples herramientas, todas con ventajas y limitaciones, como son: efectividad, manejabilidad, hemólisis mecánica y embolización distal. Las principales determinantes del éxito clínico de la intervención serán el tiempo de evolución del cuadro clínico y la realización del procedimiento invasivo. El beneficio será menor en pacientes con enfermedad tromboembólica crónica. $6,16,18$

\section{Trombectomía por aspiración}

En 1969 se introdujo el catéter de succión Greenfield, único dispositivo aprobado por la Food and Drug Administration (FDA, por sus siglas en inglés) para el tratamiento de TEP aguda (figura 3).
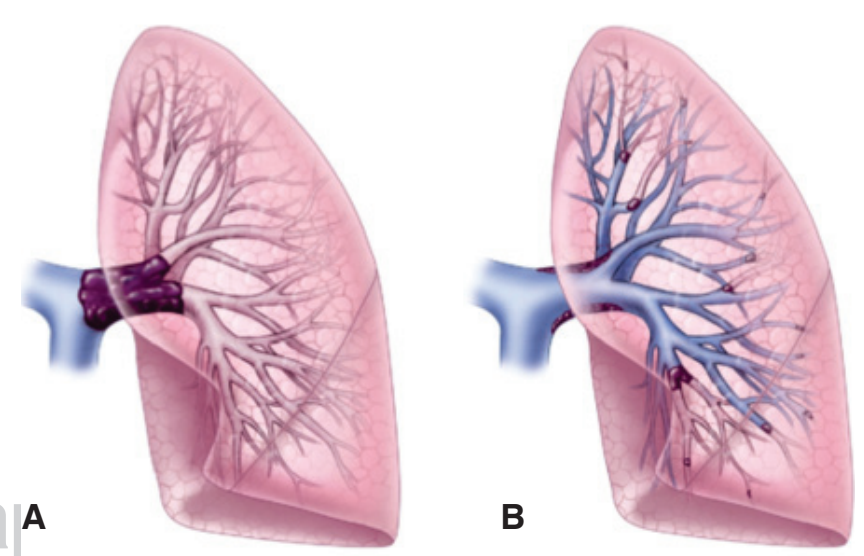

Figura 2. Esquema con trombo en arteria pulmonar izquierda principal. A) previo a la intervención asistida por catéter, en la cual se muestra disminución en la perfusión pulmonar; B) posterior a la intervención asistida por catéter, con embolización distal incrementando perfusión pulmonar.

Modificado de: Kuo W. J Vasc Interv Radiol 2012;23:167-179. (Referencia 22). 


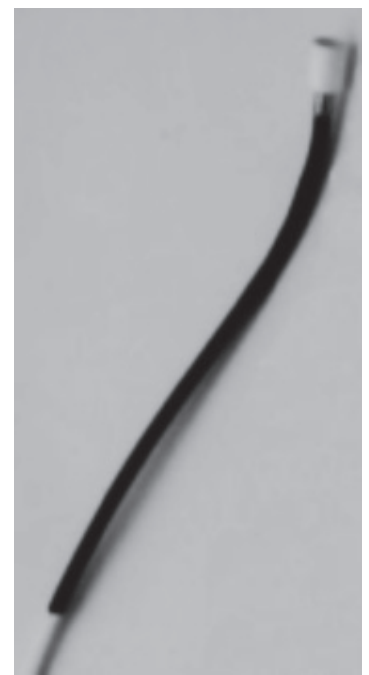

Figura 3.

Catéter de aspiración Greenfield $10 f r$.

Modificado de: Kucher N. Chest 2007;132:657-663. (Referencia 16).

Consiste de un catéter de $10 \mathrm{fr}$ con una capa de succión de 5 a $7 \mathrm{~mm}$. La mayor desventaja es que requiere de venotomía vía femoral o yugular sin guía, y el dispositivo deberá ser extraído junto con el trombo como unidad a través de venotomía quirúrgica. ${ }^{17-19}$ Este dispositivo fue evaluado por Greenfield et al., quienes mostraron su eficacia en la extracción de trombo pulmonar en cerca del $76 \%$ de los pacientes (35 de 46 pacientes), con una tasa de supervivencia a 30 días del $70 \%$. $^{12,16}$

\section{Trombectomía por fragmentación}

La fragmentación del trombo con el catéter-balón de angioplastia o con el catéter rotatorio de "cola de cochino" también se ha empleado con éxito en pequeñas series. La mayoría de los reportes del uso de estos dispositivos incluyen trombólisis local; por tanto, la eficacia de la fragmentación del trombo sin trombólisis no es clara. ${ }^{20,21}$

El principio de acción del dispositivo a través de una guía fija funciona como un eje alrededor del cual gira el catéter con el fin de fragmentar el trombo, éste embolizará de manera distal hacia la circulación pulmonar produciendo una disminución importante en la impedancia del vaciamiento del ventrículo derecho. ${ }^{22,23} \mathrm{En}$ 20 pacientes en quienes fue evaluado este dispositivo con TEP masiva, se logró una tasa de recanalización del $33 \%$ y supervivencia del $80 \%$. Se demostró una mayor efectividad cuando se utilizó de manera concomitante con terapia trombolítica ${ }^{16-24}$ (figura 4).

\section{Trombectomía hidrodinámica}

Ninguno de los dispositivos para trombectomía hidrodinámica o reolítico han sido diseñados para el tratamiento de arterias pulmonares de gran calibre. ${ }^{16}$

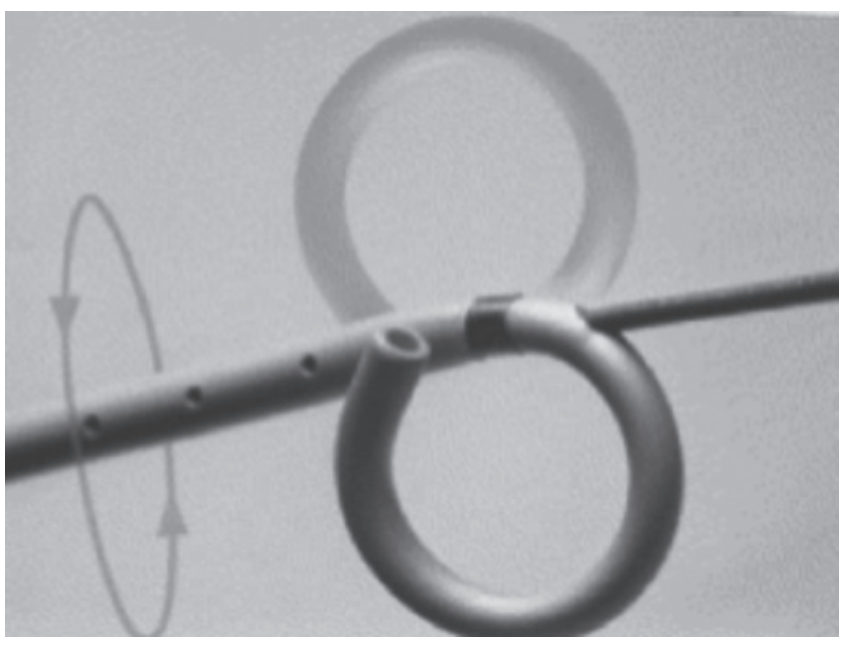

Figura 4. Catéter de trombectomía asistida para fragmentación tipo rotatorio «cola de cochino».

Modificado de: Kuo W. J Vasc Interv Radiol 2012;23:167-179. (Referencia 22).

El catéter Hydrolyzer es de 7 fr y $80 \mathrm{~cm}$, con lumen en el centro de la punta distal a través del cual se pueden aspirar los fragmentos de coágulos y sangre, y con un lumen de menor diámetro que funciona para la inyección de solución salina. La inyección a gran velocidad a través del lumen menor genera gradientes de baja presión en el lumen de mayor diámetro y un vórtex, produciendo de esta manera fragmentación y aspiración de los trombos mediante un gradiente de presión. Este dispositivo es efectivo en vasos pulmonares de $9 \mathrm{~mm}$ de diámetro. ${ }^{12,16}$

El catéter que se utiliza y tiene mayor eficacia entre los dispositivos de hidrodinamia es el AngioJet de $6 \mathrm{fr}$ con guía. No es efectivo para vasos de $>12 \mathrm{~mm}$ de diámetro. ${ }^{16}$ La principal desventaja de este sistema es que requiere de venotomía extendida para la inserción del catéter, incrementando el riesgo de sangrado en el sitio de inserción. Estos sistemas son de eficacia limitada en el tratamiento de TEP masiva. ${ }^{12,16,25}$

\section{Trombólisis asistida por catéter}

Se refiere a la infusión de algún agente trombolítico directamente al trombo en el vaso mediante un catéter multilumen guiado por imagen. ${ }^{8}$ Esta estrategia es más efectiva en eventos recientes (10-14 días) del inicio de los síntomas. ${ }^{8}$ En general, se ha documentado el éxito de esta intervención con una resolución de síntomas a cinco años en un $78 \%$ de los pacientes que reciben trombólisis asistida por catéter con anticoagulación, en 

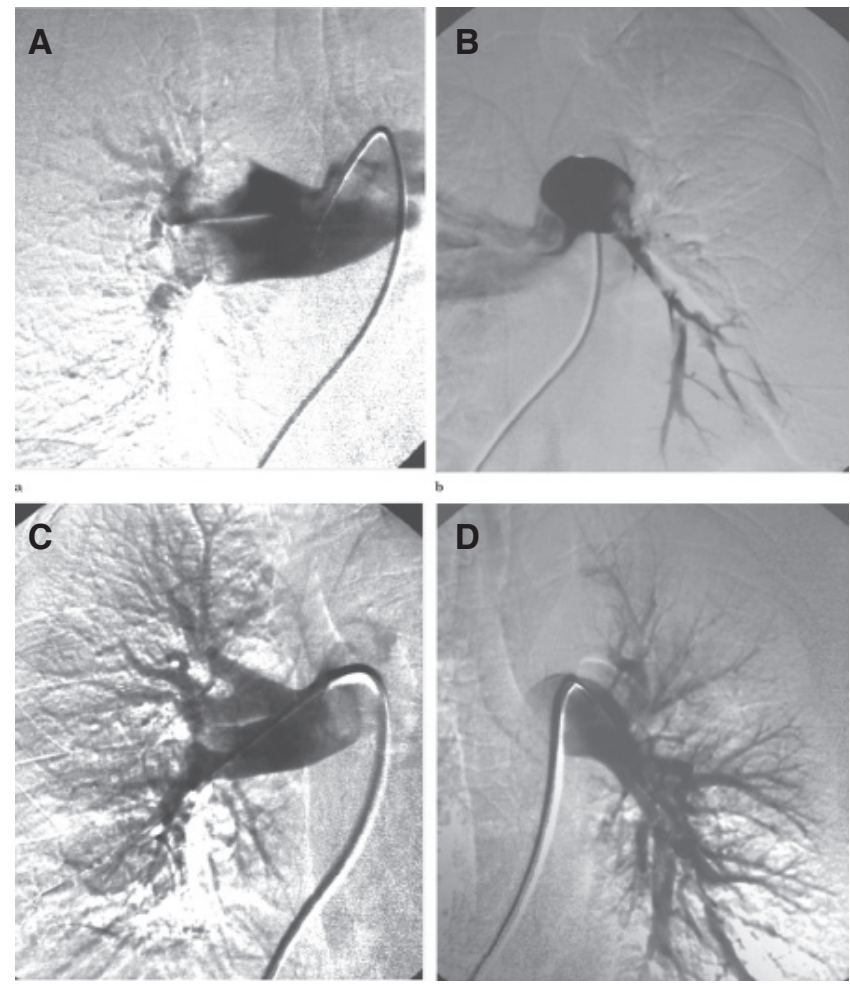

Figura 5. Angiografía pulmonar en paciente de 57 años con TEP masiva bilateral. A) angiografía pulmonar derecha inicial; B) angiografía pulmonar inicial izquierda; C y D) imágenes posteriores a trombectomía por aspiración y posterior a la inyección de agente trombolítico.

Modificado de: Kuo WT. J Vasc Interv Radiol 2012;23:167-179. (Referencia 22).

comparación con aquellos pacientes con anticoagulación únicamente $(30 \%)(p=0.0015){ }^{8}$

Existen varios reportes y estudios sobre la utilidad de este método. Consiste en administrar dosis bajas de agente trombolítico en la arteria pulmonar afectada con el fin de acelerar la destrucción del trombo y restablecer la perfusión de las arterias pulmonares ${ }^{8}$ (figura 5).

Los esquemas trombolíticos se han utilizado en combinación con HNF en pacientes con TEP masiva, ${ }^{8-12}$ tabla 3.

\section{Complicaciones asociadas a catéteres}

Son excepcionales, pero no menos importantes. Las más graves son el tamponade pericárdico y la hemorragia pulmonar. ${ }^{5}$

Comúnmente son consecuencia de la perforación o disección de alguna rama mayor de la arteria pulmonar que pueda condicionar hemorragia pulmonar masiva, $y$, por consiguiente, muerte inmediata. El riesgo de
Tabla 3. Agentes trombolíticos utilizados en combinación con HNF en TEP masiva.

\begin{tabular}{ll}
\hline Urokinasa & $250,000 \mathrm{Ul} / \mathrm{h}$ infusión para dos $\mathrm{h}$ \\
& $100,000 \mathrm{Ul} / \mathrm{h}$ para $12-24 \mathrm{~h}$ \\
Alteplase & Bolo:10 $\mathrm{mg}$ \\
& Infusión: $20 \mathrm{mg} / \mathrm{h}$ para 2 horas o 100 \\
& mg para $7 \mathrm{~h}$ \\
Reteplase & $2.5-5 \mathrm{UI}$ \\
Tenecteplase & $5-10 \mathrm{MG}$
\end{tabular}

Abreviaturas: HNF: Heparina no fraccionada; TEP: Tromboembolia pulmonar.

perforación se incrementa en vasos menores a $6 \mathrm{~mm}$. La tasa de sangrado mayor se ha reportado en $17 \%$. Con el fin de disminuir los riesgos se ha determinado, en opinión de experto, limitar la intervención terapéutica únicamente en vasos de arterias pulmonares principales y lobares, evitando arterias pulmonares segmentarias. Se deberá limitar el procedimiento una vez lograda la mejoría hemodinámica, independientemente del resultado por angiografía. ${ }^{5,8}$

También se han documentado complicaciones asociadas a los dispositivos, como las arritmias cardíacas (cuando los catéteres avanzan a través del ventrículo derecho), hemólisis mecánica e incluso pancreatitis. ${ }^{8,26}$ Otras complicaciones reportadas y relacionadas al procedimiento son: sangrado por sobreanticoagulación, nefropatía por contraste, reacciones anafilácticas al medio de contraste, complicaciones en sitio de acceso vascular como hematomas, pseudoaneurismas y fístulas arteriovenosas. , $, 8,12^{2}$

\section{CONCLUSIÓN}

El tratamiento intervencionista para TEP y TVP, a pesar de tener menor evidencia clínica en comparación con la anticoagulación, es actualmente una opción más, en especial para el tratamiento de TEP masiva. Es preciso poner a consideración cada una de estas opciones, siempre teniendo presente que se deberá elegir la ideal con base en el contexto del entorno y los recursos médicos, basados principalmente en la condición clínica del paciente. Se requieren de más estudios clínicos para validar el uso de estas maniobras terapéuticas.

\section{REFERENCIAS}

1. Horlander KT, Mannino DM, Leeper KV. Pulmonary embolism mortality in the United States, 1979-1998: an analysis using multiple-cause mortality data. Arch Intern Med 2003;163:1711-1717. 
2. Konstantinides S. Clinical acute pulmonary embolism. N Engl J Med 2008;359:2804-2813.

3. Torbicki A, Perrier A, Konstantinides S, et al. Guidelines on the diagnosis and management of acute pulmonary embolism: the Task Force for the Diagnosis and Management of Acute Pulmonary Embolism of the European Society of Cardiology (ESC). Eur Heart J 2008;29:2276-2315.

4. Goldhaber SZ, Bounameaux H. Pulmonary embolism and deep vein thrombosis. Lancet 2012;379:1835-1846.

5. Uflacker R. Interventional therapy for pulmonary embolism. J Vasc Interv Radiol 2001;12:147-164.

6. Kasper W, Konstantinides S, Geibel A, et al. Management strategies and determinants of outcome in acute major pulmonary embolism: results of a multicenter registry. J Am Coll Cardiol 1997;30:1165-1171.

7. Chung J, Owen RJ. Using inferior vena cava filters to prevent pulmonary embolism. Can Fam Physician 2008;54:49e-55e.

8. Jaff MR, McMurtry MS, Archer LA, et al. Management of massive and submassive pulmonary embolism, iliofemoral deep vein thrombosis, and chronic thromboembolic pulmonary hypertension: a scientific statement from the American Heart Association. Circulation 2011;123:1788830.

9. White RH, Zhou H, Kim J, Romano PS. A populationbased study of the effectiveness of inferior vena cava filter use among patients with venous thromboembolism. Arch Intern Med 2000;160:2033-2041.

10. Kucher N, Rossi E, De Rosa M, Goldhaber SZ. Massive pulmonary embolism. Circulation 2006;113:577-582.

11. Guyatt GH, Akl EA, Crowther M, Gutterman DD, Schuünemann HJ; American College of Chest Physicians Antithrombotic Therapy and Prevention of Thrombosis Panel. Executive summary: Antithrombotic Therapy and Prevention of Thrombosis, 9th ed: American College of Chest Physicians Evidence-Based Clinical Practice Guidelines. Chest 2012;141(2 Suppl):7S-47S.

12. Tapson VF. Interventional therapies for venous thromboembolism: vena cava interruption, surgical embolectomy, and catheter-directed interventions. Clin Chest Med 2010;31:771-781.

13. Decousus $\mathrm{H}$, Leizorovicz $\mathrm{A}$, Parent $\mathrm{F}$, et al. A clinical trial of vena cava filters in the prevention of pulmonary embolism in patients with proximal deep-vein thrombosis: Prévention du Risque d'Embolie Pulmonaire par Interruption Cave Study Group. N Engl J Med 1998;338:409-415.

14. Gulba DC, Schmid C, Borst HG, Lichtlen P, Dietz R, Luft FC. Medical compared with surgical treatment for massive pulmonary embolism. Lancet 1994;343:576-577.
15. Aklog L, Williams CS, Byrne JG, Goldhaber SZ. Acute pulmonary embolectomy: a contemporary approach. Circulation 2002;105:1416-1419.

16. Kucher N. Catheter embolectomy for acute pulmonary embolism. Chest 2007;132:657-663.

17. Verstraete $\mathrm{M}$, Miller $\mathrm{GA}$, Bounameaux $\mathrm{H}$, et al. Intravenous and intrapulmonary recombinant tissue-type plasminogen activator in the treatment of acute massive pulmonary embolism. Circulation 1988;77:353-360.

18. Eid-Lidt G, Gaspar J, Sandoval J, et al. Combined clot fragmentation and aspiration in patients with acute pulmonary embolism. Chest 2008;134:54-60.

19. Greenfield LJ, Proctor MC, Williams DM, Wakefield TW. Long-term experience with transvenous catheter pulmonary embolectomy. J Vasc Surg 1993;18:450-457.

20. Schmitz-Rode T, Janssens U, Duda SH, Erley CM, Günther RW. Massive pulmonary embolism: percutaneous emergency treatment by pigtail rotation catheter. J Am Coll Cardiol 2000;36:375-380.

21. Kuo WT, van den Bosch MA, Hofmann LV, Louie JD, Kothary N, Sze DY. Catheter-directed embolectomy, fragmentation, and thrombolysis for the treatment of massive pulmonary embolism after failure of systemic thrombolysis. Chest 2008;134:250-254.

22. Kuo WT. Endovascular therapy for acute pulmonary embolism. J Vasc Interv Radiol 2012;23:167-179.

23. Kucher N, Goldhaber SZ. Management of massive pulmonary embolism. Circulation 2005;112:e28-e32.

24. De Gregorio MA, Gimeno MJ, Mainar A, et al. Mechanical and enzymatic thrombolysis for massive pulmonary embolism. J Vasc Interv Radiol 2002;13(2 Pt 1):163-169.

25. Kucher N, Windecker S, Banz Y, et al. Percutaneous catheter thrombectomy device for acute pulmonary embolism: in vitro and in vivo testing. Radiology 2005;236:852-858.

26. Danetz JS, McLafferty RB, Ayerdi J, et al. Pancreatitis caused by rheolytic thrombolysis: an unexpected complication. J Vasc Interv Radiol 2004;15: 857-860.

\section{$\triangle$ Correspondencia:}

Dr. Jaime Eduardo Morales Blanhir

Instituto Nacional de Ciencias Médicas y Nutrición Salvador Zubirán.

Vasco de Quiroga Núm. 15, colonia Sección XVI.

México, D.F. 14000

Director Médico FEMEXER

Correo electrónico: moralesjaime@usa.net

Los autores declaran no tener conflictos de interés. 